\title{
Malignant Mesothelioma in Body Fluids - with Special Reference to Differential Diagnosis from Metastatic Adenocarcinoma -
}

Jin Ho Paik ${ }^{1,2 *} \cdot$ Jin-Haeng Chung ${ }^{1,2,4 *}$ Baek-Hui Kim ${ }^{3}$. Gheeyoung Choe ${ }^{1,4}$

'Department of Pathology, Seoul National University College of Medicine, Seoul; ${ }^{2}$ Tumor Immunity Medical Research Center at Seoul National University College of Medicine, Seoul; ${ }^{3}$ Department of Pathology, Korea University Guro Hospital, Seoul; ;Department of Pathology, Seoul National University Bundang Hospital, Seongnam, Korea

*These two authors contributed equally to this paper.

Received : June 19, 2009

Accepted : October 8, 2009

\section{Corresponding Author}

Gheeyoung Choe, M.D.

Department of Pathology, Seoul National University

Bundang Hospital, 300 Gumi-dong, Bundang-gu,

Seongnam 463-707, Korea

Tel: 031-787-7711

Fax: 031-787-4012

E-mail: gychoe@snu.ac.kr

*This study was supported by a grant of the Korea Healthcare technology R\&D project, Ministry of Health \& Welfare, Republic of Korea (A080844) and partly supported by the Korean Science \& Enginnering Foundation (KOSEF) through the Tumor Immunity Medical Research Center at Seoul National University College of Medicine. The authors are deeply gratefu to Ms. Hong Hee Joo (Taegu Foreign Language High School), for her critical proof in English.
Background : Malignant mesothelioma (MM) is a rare malignant neoplasm occurring in pleura, pericardium, and peritoneum. The differential diagnosis between MM and metastatic adenocarcinoma (MA) causes diagnostic, staging, and therapeutic dilemmas. Herein, we investigated characteristic cytologic features of MM. Methods : Cytologic specimens of MM $(n=10)$, MA $(n=25)$, and reactive mesothelial hyperplasia $(n=10)$ were retrieved and reviewed from archival materials in the Department of Pathology, Seoul National University Bundang Hospital from May 2003 to July 2008. Results : MM showed tumor cell clusters and singly scattered malignant tumor cells forming single cell populations with sparse reactive benign mesothelial cells. In contrast, MA showed distinct two cell populations of tumor cell clusters and scattered reactive mesothelial cells. Furthermore, MM frequently exhibited a characteristic long chain-like arrangement (hand-in-hand appearance) and intercellular windows, which were rarely evident in MA. Variable nuclear size, relatively consistent nuclear-cytoplasmic ratio, bior multi-nucleation, and lacy cytoplasmic borders were also frequently observed in MM. Conclusions : Differential diagnosis of MM from MA in body fluids is possible based on meticulous examination of certain cytologic parameters, which could have significant implications in staging and treatment.

Key Words : Cytology; Mesothelioma; Adenocarcinoma; Body fluids; Diagnosis, Differential
Malignant mesothelioma (MM) is a rare malignant tumor occurring in pleura, peritoneum, and pericardium. Similarities with metastatic adenocarcinoma (MA) can occasionally hamper differential diagnosis as well as cause diagnostic, staging and therapeutic dilemmas in clinical management. Since the two tumors have different origins, primary sites, staging systems and therapeutic options, the diagnosis of body fluid involvement would differentially affect therapeutic strategies or prognosis of patients. From the view point of pathologists, the two tumors are occasionally confused even in biopsy material, requiring ancillary tests such as immunohistochemistry and electron microscopy, which add to the time and cost of diagnosis. ${ }^{1}$ A number of trials have sought to differentiate between MM and MA on histologic sections using panels of multiple immunohistochemical 
markers and complex statistical analysis, ${ }^{1-3}$ exemplifying the difficulty in differentiating MM from MA. Sometimes, even histology supported by immunohistochemistry may not conclusively distinguish the two tumors. In contrast, body fluid cytology is superior to histology in observing delicate cytomorphologic details, and represents a less invasive, inexpensive, simple, and quick means of diagnosis. Body fluid is often the first and sometimes the only specimen submitted to pathologists, which requires more attention to cytologic diagnosis. Therefore, precise distinction between MM and MA on the basis of body fluid cytology according to reasonable cytomorphologic criteria is not only important and useful, but also cost- and timeeffective. The reality of practical pathologic examinations includes occasional cases where careful morphologic examination with appropriate parameters or methods is more useful to reach the conclusion, avoiding unnecessary procedures to patients. ${ }^{4}$ In that context, the aim of this study was to define characteristic cytologic features of MM distinct from those of MA, and investigate the practical usefulness of the cytologic parameters.

\section{MATERIALS AND METHODS}

\section{Patients and samples}

Cytologic specimens of $10 \mathrm{MM}, 25 \mathrm{MA}$, and 10 reactive mesothelial hyperplasias were retrieved from the archival materials in the Department of Pathology, Seoul National Universi- ty Bundang Hospital from May 2003 to July 2008. The diagnosis of MM was supplemented by immunostaining on histologic sections or cell blocks in nine cases, or by histology alone in one case. The specimens were from eight male and two female patients diagnosed with MM (39-82-years-of-age, mean age 64.6 years; Table 1). Body fluid samples were pleural fluid in seven cases, peritoneal fluid in two cases, and pericardial fluid in one case. The diagnosis of MM was based on the criteria in the World Health Organization classification of lung and pleural tumors. ${ }^{5}$ Primary sites of $25 \mathrm{MA}$ were the lung in 12 cases, stomach in seven cases, bile duct in two cases, and one case each from colon, breast, pancreas, and ovary. All 25 cases were pleural fluids obtained from nine males and 16 females (28-79-yearsof-age, mean age 55.7 years). All 10 reactive mesothelial hyperplasia samples were pleural fluids.

\section{Cytology interpretation and statistical analysis}

Papanicolaou and Diff-Quik staining was performed after cytocentrifugation of all body fluid specimens. Cytologic evaluation was performed with special reference to several cytomorphologic criteria including: 1) cellular composition containing scattered and clustered atypical cells, 2) long chain-like arrangement, 3) intercellular window formation, 4) fuzzy margin with microvilli, 5) intracytoplasmic vacuole formation, 6) infiltration of inflammatory cells, and 7) other conventional cytologic features described for MM or MA. Cytology slides were blindly reviewed by three pathologists (B.H.K., J.H.C., and J.H.P.) and

Table 1. Clinical and pathologic features of 10 malignant mesothelioma cases

\begin{tabular}{|c|c|c|c|c|c|c|c|c|}
\hline \multirow[b]{2}{*}{$\begin{array}{c}\text { Case } \\
\text { no. }\end{array}$} & \multirow[b]{2}{*}{ Age } & \multirow[b]{2}{*}{ Sex } & \multirow[b]{2}{*}{$\begin{array}{l}\text { Primary } \\
\text { site }\end{array}$} & \multicolumn{3}{|c|}{ Additional diagnostic modalities } & \multirow[b]{2}{*}{$\begin{array}{l}\text { Histologic } \\
\text { type }\end{array}$} & \multirow[b]{2}{*}{ Main immunostaining findings } \\
\hline & & & & Histology & $\begin{array}{c}\text { IHC on } \\
\text { histologic } \\
\text { sections }\end{array}$ & $\begin{array}{l}\text { ICC } \\
\text { on } \\
\text { CB }\end{array}$ & & \\
\hline 1 & M & 63 & Pleura & No & No & Yes & NA & $\mathrm{CK}+, \mathrm{VT}+, \mathrm{HBME}-1_{+}$, Calretinin $\mathrm{f}_{+}, \mathrm{CEA} \mathrm{f}_{+}, \mathrm{CK} 5 / 6+$ \\
\hline 2 & $\mathrm{~F}$ & 59 & Peritoneum & Yes & Yes & No & NA & CK+, VT+, Calretinin+, HBME-1+, CEA-, LeuM-1- \\
\hline 3 & M & 39 & Pericardium & Yes & Yes & No & Biphasic & $\mathrm{CK}+, \mathrm{VT}+, \mathrm{D} 2-40+$, Calretinin,$+ \mathrm{CK} 5 / 6 \mathrm{f}+$ \\
\hline 4 & M & 71 & Pleura & Yes & Yes & Yes & NA & $\mathrm{CK}+, \mathrm{VT}+$, Calretinin+, D2-40-, TTF-1-, CEA- \\
\hline 5 & M & 61 & Peritoneum & Yes & Yes & No & Epithelioid & CK5/6+, VT+, Calretinin+, D2-40+, CEA- \\
\hline 6 & M & 68 & Pleura & Yes & Yes & Yes & Biphasic & $\begin{array}{l}\text { CK+, VT+, CK5/6 w+, Calretinin+, D2-40 f+, HBME-1-, } \\
\text { TTF-1 f+, CEA-, LeuM-1- }\end{array}$ \\
\hline 7 & M & 68 & Pleura & Yes & Yes & Yes & Biphasic & $\mathrm{CK}+, \mathrm{VT}+, \mathrm{CK} 5 / 6+, \mathrm{CEA} \mathrm{f}_{+}$, Calretinin,$+ \mathrm{HBME}-1+$ \\
\hline 8 & M & 59 & Pleura & Yes & No & No & $\begin{array}{c}\text { Well } \\
\text { differentiated } \\
\text { papillary }\end{array}$ & NA \\
\hline 9 & $\mathrm{~F}$ & 82 & Pleura & No & No & Yes & NA & Calretinin+, D2-40+, HBME-1+, CEA-, TTF-1- \\
\hline 10 & M & 76 & Pleura & No & No & Yes & Epithelioid & Calretinin,$+ \mathrm{CK} 5 / 6+$ \\
\hline
\end{tabular}

$\mathrm{NA}$, not analyzable; IHC, immunohistochemistry; ICC, immunocytochemistry; CB, cell block; CK, cytokeratin; VT, vimentin; CEA, carcinoembryonic antigen; TTF-1, thyroid transcription factor-1; +, positive; -, negative; $f+$, focal positive; $w+$, weak positive. 
cytologic findings were categorized as present, absent, or equivocal as for the aforementioned specific items. To make the descriptive terms clear and objective, we used the terms, "two-dimensional (2D) cell group" and "three-dimensional (3D) cell group" instead of "papilla" or "cell ball," respectively, reflecting only the presence of 2D and 3D structures, regardless of shape. Moreover, to reflect the proportion of cell groups, especially in cases having both 2D and 3D cell groups, we evaluated the "major component" between these groups, which was defined as cell groups accounting for $>50 \%$ of observed total cell groups. The cases where the extent of 2D and 3D cell groups were similar or cases where cell group formation was poor and consisted mainly of scattered tumor cells were categorized as equivocal. Histology and immunostaining of cell block or histologic sections were compared to cytologic features of body fluid samples. For statistical analysis, Pearson's chi-square test was done using SAS version 8.01 (SAS Institute, Cary, NC, USA) for a specific finding in MA and MM, regarding a p-value $<0.05$ as significant. Although the same cytologic findings used in differentiation between MM and MA were applied to reactive mesothelial hyperplasia in Table 2, the degree of atypism was not obviously related to the malignant level of MM or MA. Reactive mesothelial hyperplasia was not confused with MM or MA in most cases, so statistical analysis was performed only between MM and MA.

\section{RESULTS}

Table 1 shows profiles of $10 \mathrm{MM}$ cases diagnosed on the bases of cytology, histology and immunostaining. Histologic types were biphasic type containing epithelioid component $(n=3)$, epithelioid type $(n=2)$, well-differentiated papillary type $(n=1)$, and 'not analyzable' $(\mathrm{n}=4)$. The latter lacked a sufficient amount of biopsy material or contained severe cautery artifact, which precluded assignment of a definite histologic types, but at least focally included an epithelioid component. Although some cases showed partially overlapping, focal, weak, lacking, or aberrant immunostaining findings in the differentiation of $\mathrm{MM}$ and MA, the collective interpretation of cytology, histology, and multiple immunostaining allowed an unequivocal diagnosis.

Next, we compared MM and MA with all the possible cytomorphologic parameters and examined whether each of the parameters were really useful for differentiation on cytologic preparation alone. As compared with MA, MM showed characteristic morphological features in terms of cellular arrangement and individual cell appearance (Fig. 1A-H, Table 2). Regarding the

Table 2. Comparison of cytologic features between malignant mesothelioma, metastatic adenocarcinoma, and reactive mesothelial hyperplasia

\begin{tabular}{|c|c|c|c|c|c|c|c|c|c|c|}
\hline \multirow{2}{*}{ Cytologic features of atypical cells } & \multicolumn{3}{|c|}{$\mathrm{MM}, \mathrm{N}=10$} & \multicolumn{3}{|c|}{$M A, N=25$} & \multicolumn{3}{|c|}{$\mathrm{RMH}, \mathrm{N}=10$} & \multirow{2}{*}{$p$-value ${ }^{a}$} \\
\hline & - & $\triangle$ & + & - & $\triangle$ & + & - & $\triangle$ & + & \\
\hline Presence of singly scattered atypical cells & 1 & 0 & 9 & 11 & 4 & 10 & 6 & 2 & 2 & $0.0057^{b}$ \\
\hline $\begin{array}{l}\text { Distinct two cell population } \\
\text { (single cells vs clusters) }\end{array}$ & 0 & 4 & 6 & 14 & 9 & 2 & 10 & 0 & 0 & $<0.0001^{\text {b }}$ \\
\hline Presence of 2D cell group & 1 & 1 & 8 & 4 & 5 & 16 & 5 & 0 & 5 & 0.5945 \\
\hline Presence of 3D cell group & 4 & 1 & 5 & 5 & 6 & 14 & 8 & 2 & 0 & 0.2625 \\
\hline Major component (2D vs 3D) & $2 \mathrm{D}: 6$ & 2 & 3D:2 & 2D:10 & 3 & 3D:12 & 2D:5 & 5 & 3D:0 & 0.0822 \\
\hline Border scalloping of cell clusters & 2 & 1 & 7 & 5 & 4 & 16 & 7 & 1 & 2 & 0.7004 \\
\hline Long chain appearance of atypical cells & 4 & 0 & 6 & 22 & 3 & 0 & 8 & 2 & 0 & $<0.0001^{\text {b }}$ \\
\hline Intercellular window & 1 & 1 & 8 & 18 & 4 & 3 & 4 & 0 & 6 & $<0.0001^{\mathrm{b}}$ \\
\hline Marked variability of nuclear size & 0 & 1 & 9 & 11 & 1 & 13 & 10 & 0 & 0 & $0.0011^{\mathrm{b}}$ \\
\hline Relatively consistent nuclear-cytoplasmic ratio & 0 & 0 & 10 & 15 & 3 & 7 & 0 & 0 & 10 & $<0.0001^{b}$ \\
\hline Bi- or multi-nucleated atypical cells & 0 & 0 & 10 & 11 & 3 & 11 & 3 & 0 & 7 & $0.0018^{b}$ \\
\hline Prominent nucleoli with vesicular chromatin & 1 & 0 & 9 & 6 & 2 & 17 & 2 & 0 & 8 & 0.2038 \\
\hline Cytoplasmic vacuoles & 0 & 2 & 8 & 8 & 2 & 15 & 4 & 0 & 6 & $0.0088^{\circ}$ \\
\hline Cell-in-cell appearance & 3 & 3 & 4 & 16 & 1 & 8 & 10 & 0 & 0 & $0.0080^{b}$ \\
\hline Fuzzy border with microvilli & 0 & 0 & 10 & 20 & 3 & 2 & 1 & 2 & 7 & $<0.0001^{\mathrm{b}}$ \\
\hline Inflammatory cells outnumbering atypical cells & 2 & 2 & 6 & 14 & 2 & 9 & 4 & 0 & 6 & $0.0055^{b}$ \\
\hline Psammoma body & 10 & 0 & 0 & 24 & 0 & 1 & 10 & 0 & 0 & 1.000 \\
\hline
\end{tabular}

aindicates $p$ value by Pearson's $\chi^{2}$-test in MM and MA, and bindicates $p<0.05$; 'means cell groups accounting for more than $50 \%$ of total observed atypical cell groups.

MM, malignant mesothelioma; MA, metastatic adenocarcinoma; RMH, reactive mesothelial hyperplasia; -, absent; $\triangle$, equivocal; +, present; 2D, two dimensional; 3D, three dimensional. 
cellular pattern, MM frequently ( $\mathrm{p}=0.0057)$ showed singly scattered tumor cells (Fig. 1A) and frequently $(\mathrm{p}<0.0001)$ displayed biphasic tumor cell composition (i.e., morphologically identi- cal malignant cells from both tumor cell clusters and adjacent scattered tumor cells; "one cell population of scattered and clusters of atypical cells" with rare intermixed reactive benign meso-

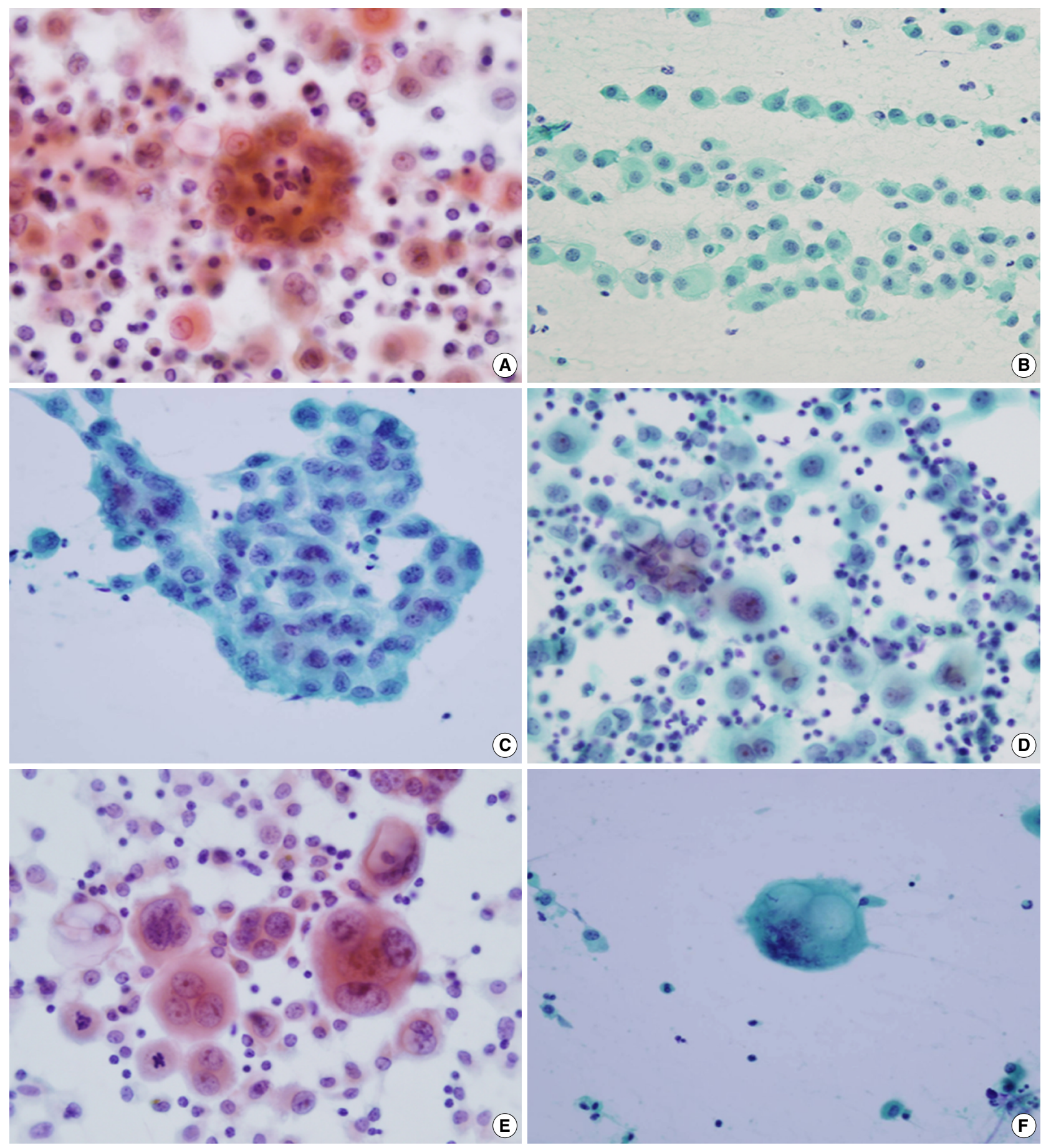

Fig. 1. Common cytologic features of malignant mesothelioma (Papanicolaou stain). (A) Singly scattered and clusters of tumor cells form one cell population are observed. (B) The tumor cells arrange in a long chain-like pattern with a "hand-in-hand" appearance. (C) Intercellular windows are observed in two-dimensional tumor cell sheets. (D) Variable sized tumor cells present a monotonous appearance with relatively consistent nuclear-cytoplasmic ratio. (E) Multinucleated cells with large nucleoli are commonly observed. (F) The cytoplasm of tumor cells shows prominent vacuoles and fuzzy margin with microvilli. (Continued to the next page) 


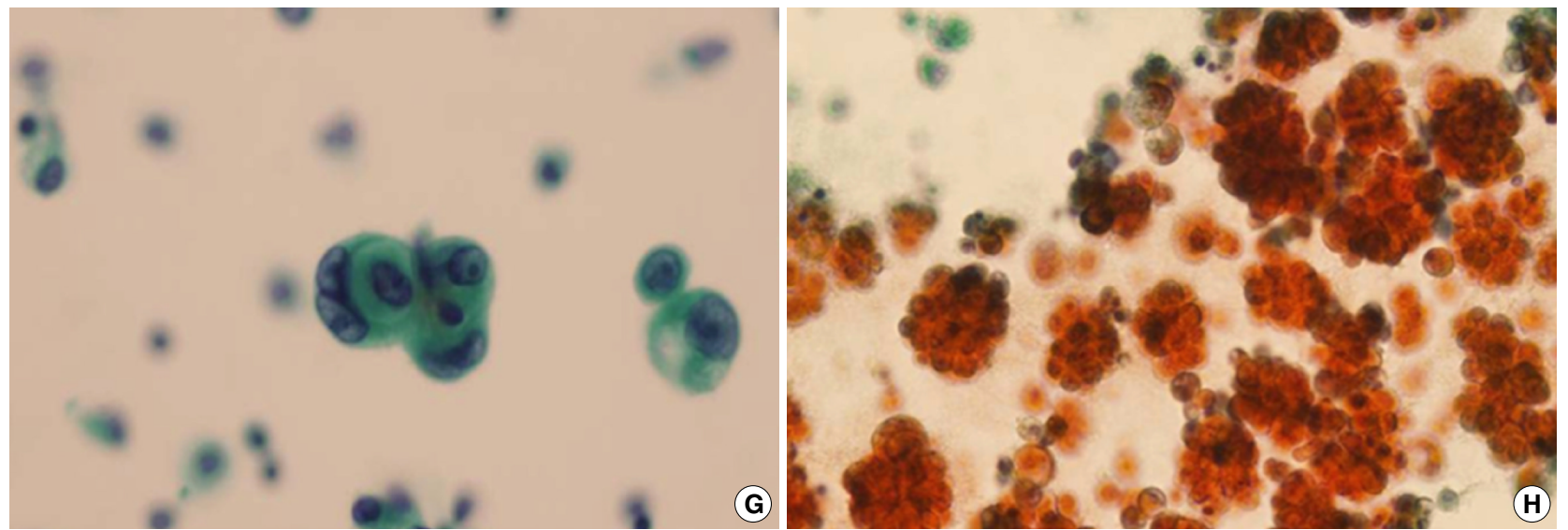

Fig. 1. (Continued from the previous page) (G) The tumor cells are overlapped forming a cell-in-cell appearance. (H) The tumor cell groups form papillary clusters with scalloped borders.
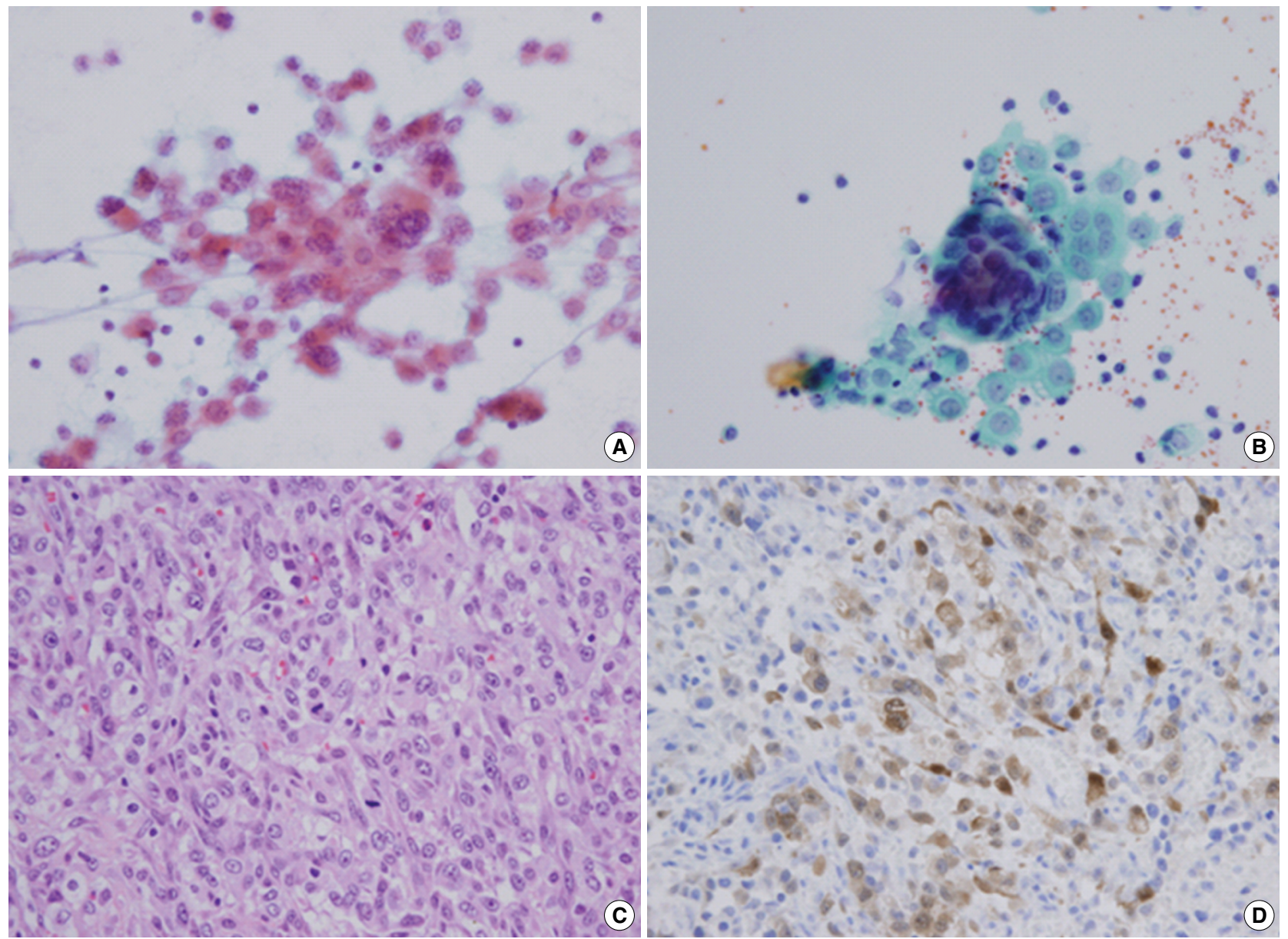

Fig. 2. Comparison of representative patterns of malignant mesothelioma and metastatic adenocarcinoma. (A) Malignant mesothelioma shows loose or less cohesive cell groups forming irregularly linear arrangement with singly scattered tumor cells of one cell population (Papanicolaou stain). (B) Metastatic adenocarcinoma cell clusters are surrounded by reactive mesothelial cells, forming two cell populations (Papanicolaou stain). (C) Histologic sections show solid sheets of malignant mesothelioma cells (H\&E stain). (D) Immunohistochemistry for calretinin shows focal positivity in malignant mesothelioma. 
thelial cells) (Fig. 1A). In contrast, MA mainly showed the "distinct two cell population" composed of overtly malignant tumor cell clusters and scattered adjacent benign mesothelial cells lacking definite atypia that could be regarded as malignant, each of which was morphologically different. Furthermore, characteristic long chain-like arrangement ("hand-in-hand appearance") (Fig. 1B) and intercellular windows (Fig. 1C) were frequently observed in $\mathrm{MM}$ ( $\mathrm{p}<0.001$ in both instances), which were absent or rare in MA.

Individual tumor cells of MM showed round-to-polygonal contours with markedly variable nuclear size (Fig. 1D; p=0.0011) but displayed a relatively consistent nuclear-cytoplasmic ratio (Fig. 1D; p<0.0001). Bi- or multi-nucleated tumor cells (Fig. $1 \mathrm{E} ; \mathrm{p}=0.0018$ ), intracytoplasmic vacuoles (Fig. 1F; $\mathrm{p}=0.0088$ ), cell-in-cell appearance (Fig. 1G; p=0.0080), lacy cytoplasmic borders with microvilli (Fig. 1F; $\mathrm{p}<0.0001$ ), and inflammatory cells outnumbering tumor cells (Fig. 1D; $\mathrm{p}=0.0055$ ) were also commonly observed in MM.

As conventionally described, 2D and 3D cell groups were compared between MM and MA. However, both cell groups were frequently observed in both MM and MA. When the major component accounting for $>50 \%$ of observed cell groups was compared in MM and MA, 2D cell groups tended to be frequently observed in MM, while 3D cell groups predominated in MA, although neither reached statistical significance $(p=0.0822)$. Other cytologic parameters including border scalloping of cell clusters (Fig. 1H), prominent nucleoli with vesicular chromatin, and psammoma bodies were not significantly different between $\mathrm{MM}$ and MA ( $\mathrm{p}>0.05)$.

Fig. 2A, B depict the distinctive features of MM and MA. The 2D cell group of $\mathrm{MM}$ was frequently observed as a loose reticular pattern, tending toward a linear arrangement or single tumor

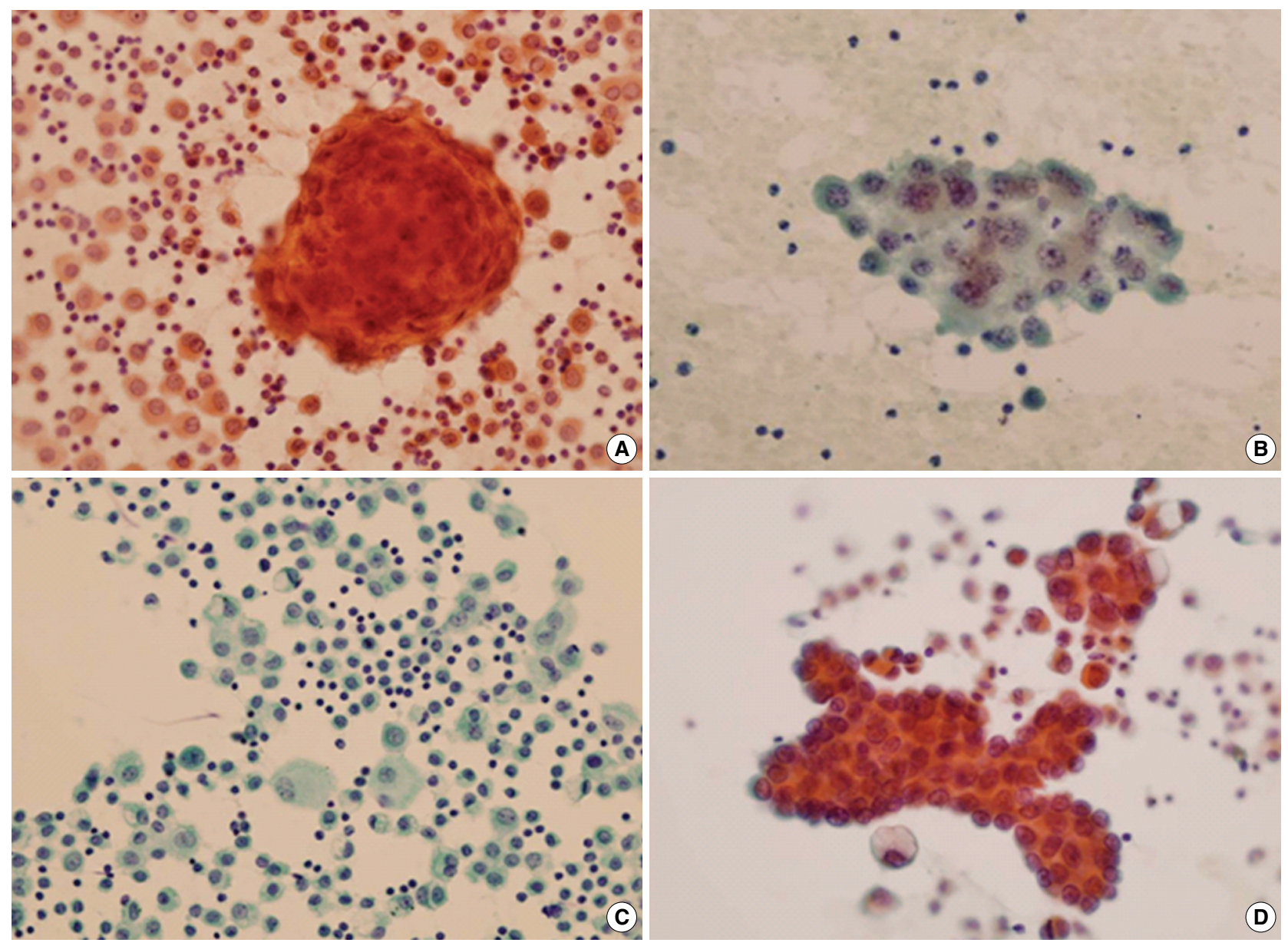

Fig. 3. Confusing cytologic features in diagnosis of malignant mesothelioma (Papanicolaou stain). (A) Malignant mesothelioma occasionally shows 3-D cell ball pattern. (B) Metastatic adenocarcinoma shows 2-D cell groups with intercellular window-like spaces. (C) Atypical mesothelial cells are scattered in reactive mesothelial hyperplasia. (D) Papillary clusters with scalloped borders are relatively common in metastatic adenocarcinoma. 
cells, which formed a single cell population with an intercellular window and relatively conserved nuclear cytoplasmic ratio despite variable nuclear size (Fig. 2A). In contrast, MA frequently revealed a distinct two cell population consisting of obvious malignant cell clusters without intercellular window surrounded by benign mesothelial cells (Fig. 2B). The diagnosis of MM was supplemented with histology and/or immunostaining (Fig. 2C, D).

Among all the examined cytologic parameters, some features were found to be significantly different, while others did not reach statistical significance in the distinction of $\mathrm{MM}$ and MA. In particular, some conventionally emphasized cytologic features were variably overlapped, which might confuse differential diagnosis without integrated analysis using the aforementioned cytologic findings. As shown in Fig. 3, 3D cell groups (conventional "cell ball formation") in MM (Fig. 3A), or 2D cell groups with intercellular window-like spaces in MA (Fig. 3B) were rarely observed. More frequently, atypical mesothelial cells were likely to be observed in reactive mesothelial hyperplasia, which lacked definite cellular atypia and complexity of arrangement (Fig. 3C). Frequently, 2D cell groups forming papillary clusters with scalloped borders were observed in MA (Fig. 3D).

\section{DISCUSSION}

$\mathrm{MM}$ is a rare malignant neoplasm accounting for $<2 \%$ of malignant tumors and $1.6 \%$ of malignant pleural effusions. ${ }^{6.7}$ It is well-known that MM is related to occupational asbestos exposure, and its latency period is more than 20 years, ${ }^{7}$ while some viral exposure with SV40 has been etiologically suggested. ${ }^{8}$ Once this rare tumor occurs in pleura, pericardium, or peritoneum, its diagnosis is challenging in many cases since distinction from other tumors can be onerous. ${ }^{9}$ In the cases of pleural or pericardial involvement, $\mathrm{MA}$ from lung is the main differential diagnosis, while peritoneal involvement should be distinguished from MA of gynecologic or gastrointestinal origin. ${ }^{10,11}$ Clinically, body cavity effusions might occasionally be an initial presentation, ${ }^{12}$ and body cavity fluid cytology is commonly submitted without sufficient information of the possibility of other malignancies, which limits cytologic diagnosis. Nevertheless, careful cytomorphologic examination has been regarded as a reliable and accurate method, ${ }^{13}$ although overall sensitivity of body fluid cytology has been variably reported. ${ }^{13,14}$ The correct detection of $\mathrm{MM}$ is critical in that positive effusion in $\mathrm{MM}$ might be in less advanced stages as compared with MA, ${ }^{7}$ which would lead to optimal treatments.
In the present study, we reviewed $10 \mathrm{MM}$ and $25 \mathrm{MA}$ body fluid cytology cases supplemented with histology or immunostaining, and we characterized cytologic features of $\mathrm{MM}$ as compared with MA. Although no single feature could completely distinguish between MM and MA and some features partially overlapped, collective consideration of general arrangement pattern combined by individual cell morphology was useful for cytological distinction between the two tumors.

Of the aforementioned cytologic features, long chain-like arrangement (hand-in-hand appearance) was uniquely observed as the powerful cytologic parameter; this pattern is suggestive of MM. ${ }^{11}$ It was observed as a linear arrangement of tumor cells containing 10 or more tumor cells, usually with loose direct contact with the adjacent tumor cells. However, two adjacent tumor cells sometimes displayed a short gap of $\leq$ one cell width, intermixed with those having loose direct contact. This latter contact was the connection of two adjacent tumor cells with minimal areas of cytoplasmic surface occasionally showing cytoplasmic protrusions or fuzzy microvilli-like borders, reminiscent of "hand-in-hand" appearance. Each tumor cell that formed a row in the long chain-like arrangement could show specific cytologic features such as mitosis, cell-in-cell appearance, cytoplasmic vacuole, bi-nucleation, and prominent nucleoli. Long chain-like arrangement was mostly shown with adjacent tumor cell arrangements forming similar structures either in a parallel or non-parallel pattern. A parallel pattern was observed as at least several (in many cases, most) arrangements of tumor cells, which were arranged mainly in parallel rows (Fig. 1B). In some cases that displayed the parallel pattern, parallel rows occasionally merged and were interconnected at some point forming loose sheared net-like or reticular patterns. A non-parallel pattern was observed as tumor cell arrangements lacking definite parallel pattern, evident mostly as linear tumor cell rows attached to the periphery of loose sheared net-like or partly torn reticular 2D structures, as a possible intermediate becoming long chain-like arrangement (Fig. 2A). On the assumption that the configuration of MM cell clusters in cytologic smear reflected a preserved nature of benign mesothelial cells, which two-dimensionally line and cover body wall, and the growth pattern of MM in body cavity that might be modified by shearing forces during cytologic preparation, the long chain-like arrangement was thought to be the dissolving form of MM cell clusters modified by shearing forces in cytologic preparation, producing unique patterns of cellular arrangement in MM. Although long chain-like pattern was observed in 60\% of MM samples, it was specific to MM, and so provided a useful discriminator. While carcinoma cells exhibit a similar linear 
arrangement, usually observed in aspiration smear preparation from lobular carcinoma of breast or other carcinomas, the arrangement could be differentiated from the long chain-like arrangement of MM, with a relatively "short chain" -like pattern and sparse density that hardly exceeded 10 cells and several chains on a smear slide. A few cases of MA did display a similar linear arrangement with non-parallel pattern in pleural fluid smears, which could not meet the length criterion.

Among the total of 10 cases of MM in the present study, the histologic types were 'not analyzable' in four cases, epithelioid type in two cases, well differentiated papillary type in one case, and biphasic type containing epithelioid component as a major component in three cases. The four 'not analyzable' cases included two cases having only cell block sections and two cases having both cell block and biopsy material in which assignment of the histological type was impeded by the lack of material or presence of severe cautery artifact. However, the contents of these four cases in cell block sections or histologic sections showed at least focally epithelioid features, which suggested the presence of epithelioid components. Therefore, all MM cases appeared to mainly contain epithelioid components, and the relationship between presence of long chain-like arrangement of tumor cells and histologic types was not significant.

Another notable differential point is homogeneity of cell population, ${ }^{11}$ which consists of tumor cell clusters and adjacent singly scattered cells. Recognition of two cell populations suggested that the tumors originated from other than the mesothelium, because singly scattered cells were mainly reactive mesothelial cells. Interestingly, in cytologic preparations of some MM cases, 2D tumor cell sheets tended to become loose sheets, long chain, or scattered single tumor cells, as a morphologic continuum, all of which were composed of one cell population (Fig. 2A).

Other conventionally well-known features also found as useful parameters included intercellular window, markedly variable nuclear size, relatively consistent nuclear-cytoplasmic ratio, bior multi-nucleated tumor cells, intracytoplasmic vacuole, cellin-cell appearance, lacy cytoplasmic borders with microvilli, and inflammatory cells outnumbering tumor cells. Among them, intercellular window, relatively consistent nuclear-cytoplasmic ratio, and lacy border with microvilli were especially useful parameters $(\mathrm{p}<0.0001)$.

In the present study, recognition of two newly described unique cytological patterns was very useful for differentiation of MM containing mainly epithelioid type and MA. These were cellular composition consisting of atypical cell groups and adjacent scattered cells (one cell pattern in MM vs two cell pattern in MA), and long chain-like arrangement mainly in MM. Although these two features themselves were not fully sufficient for distinction of MM and MA, the integrated analysis of various cytologic characteristics was powerful for differentiation of MM and MA. For the integrated analysis on the basis of morphology, recognition of specific and easily discernable patterns is important and can practically be the basis for extension to other cytologic features. In that context, this study has significant implications for not only cytomorphologic basis of "pattern recognition" but also clinical and therapeutic managements.

There was a well-differentiated papillary mesothelioma (WDPM) of pleura in our series, which is very rare, especially in pleura, and which displays an indolent clinical course unlike conventional MM. ${ }^{15}$ As compared with the other nine MM cases, WDPM showed relatively abundant round-shape papillary cell groups covering large portions of the slide surface, with a less pleomorphic nuclear feature. Although WDPM also tended to form a one cell pattern like other MM cases, its relatively bland-looking scattered tumor cells were not strikingly distinct from intermixed reactive mesothelial cells, which required careful interpretation in recognizing "patterns."

Immunostaining on histologic sections or cell block generally distinguishes between MM and MA, especially with pancytokeratin, vimentin, cytokeratin 5/6, calretinin, D2-40, TTF-1 and CEA. ${ }^{11,16,17}$ However, not infrequently, the immunostaining pattern can overlap between MM and MA, or is weak, focal, aberrant or lacking, which can confuse differential diagnosis without cytomorphologic consideration. Immunostaining results using specific antibody vary widely in lung cancer according to different antibody clones, ${ }^{18}$ which requires more cautious interpretation.

In summary, differential diagnosis of MM from MA in body fluid was possible based on certain cytologic parameters and can be even more helpful than histopathologic diagnosis when a scant amount of biopsy material is available and/or in the presence of cautery artifact, which can complicate immunoprofiling. Therefore, meticulous examination using the panel of cytologic parameters elucidated in this study would lead to early and accurate diagnosis of $\mathrm{MM}$, avoiding misdiagnosis and inappropriate invasive procedures.

\section{REFERENCES}

1. Yaziji H, Battifora H, Barry TS, et al. Evaluation of 12 antibodies for distinguishing epithelioid mesothelioma from adenocarcinoma: 
identification of a three-antibody immunohistochemical panel with maximal sensitivity and specificity. Mod Pathol 2006; 19: 514-23.

2. Soini Y, Kinnula V, Kahlos K, Paakko P. Claudins in differential diagnosis between mesothelioma and metastatic adenocarcinoma of the pleura. J Clin Pathol 2006; 59: 250-4.

3. Klebe S, Nurminen M, Leigh J, Henderson DW. Diagnosis of epithelial mesothelioma using tree-based regression analysis and a minimal panel of antibodies. Pathology 2009; 41: 140-8.

4. Xu X, Chung JH, Jheon S, et al. The accuracy of frozen section diagnosis of pulmonary nodules: evaluation of inflation method during intraoperative pathology consultation with frozen section. J Thorac Oncol 2009 (In Press).

5. Travis WD, Brambilla E, Muller-Hermelink HK, Harris CC. World Health Organization classification of tumours. Pathology and genetics of tumours of the lung, pleura, thymus and heart. Lyon: IARC Press, 2004; 128-36.

6. Pedio G, Landolt-Weber U. Cytologic presentation of malignant mesothelioma in pleural effusions. Exp Cell Biol 1988; 56: 211-6.

7. Greene F, Page D, Fleming I, et al. AJCC cancer staging manual. 6th ed. New York: Springer-Verlag, 2002; 167-84.

8. Zervos MD, Bizekis C, Pass HI. Malignant mesothelioma 2008. Curr Opin Pulm Med 2008; 14: 303-9.

9. Duggan MA, Powers CN. Body cavity fluid cytology. Pathol Case Rev 2006; 11: 65-6.
10. Jain R. Pelvic washings and the staging of gynecologic cancers. Pathol Case Rev 2006; 11: 92-7.

11. Bhatti T, Tabbara S. Malignant mesothelioma: fluid cytology and differential diagnostic features. Pathol Case Rev 2006; 11: 67-73.

12. Filie A, Jones H. Metastatic carcinoma in effusions: Query primary site. Pathol Case Rev 2006; 11: 74-7.

13. Malamou-Mitsi VD, Zioga AP, Agnantis NJ. Diagnostic accuracy of pericardial fluid cytology: an analysis of 53 specimens from 44 consecutive patients. Diagn Cytopathol 1996; 15: 197-204.

14. Renshaw A, Dean B, Antman K, Sugarbaker DJ, Cibas ES. The role of cytologic evaluation of pleural fluid in the diagnosis of malignant mesothelioma. Chest 1997; 111: 106-9.

15. Kao S, Mahon K, Lin B, et al. Pleural well-differentiated papillary mesothelioma: a case report. J Thorac Oncol 2009; 4: 920-2.

16. Muller AM, Franke FE, Muller KM. D2-40: a reliable marker in the diagnosis of pleural mesothelioma. Pathobiology 2006; 73: 50-4.

17. Mimura T, Ito A, Sakuma T, et al. Novel marker D2-40, combined with calretinin, CEA, and TTF-1: an optimal set of immunodiagnostic markers for pleural mesothelioma. Cancer 2007; 109: 933-8.

18. Lee HJ, Xu X, Choe G, et al. Protein overexpression and gene amplification of epidermal growth factor receptor in nonsmall lung carcinomas: comparison of four commercially available antibodies by immunohistochemistry and fluorescence in situ hybridization study. Lung Cancer 2009 (In Press). 